\title{
Efecto de la práctica de actividad física gamificada en el estado de ánimo de jugadoras de baloncesto en etapa de confinamiento Effect of gamified physical activity practice on the mood of female basketball players in confinement stage \\ Alberto Rodríguez Cayetano, Estela Vicente Rivera, José Manuel De Mena Ramos, Salvador Pérez Muñoz Universidad Pontificia de Salamanca (España)
}

Resumen. El objetivo principal de esta investigación es analizar el efecto en el estado de ánimo de jugadoras de baloncesto, a través de la práctica de actividad física gamificada durante la etapa de confinamiento debido a la COVID-19. La muestra utilizada fue de 26 jugadoras de tres categorías diferentes: infantil, juvenil y senior, con una media de edad de 16.65 ( \pm 3.84 ) años. Para analizar el efecto sobre el estado de ánimo, se realizó un estudio cuasi-experimental a través de un estudio con un pretest, una intervención y un postest final, proporcionando el cuestionario de Profile of Mood States (POMS) antes y después de cada sesión. Los resultados obtenidos mostraron mejoras en el estado de ánimo, disminuyendo los valores que se relacionan con estados emocionales negativos. Durante etapas de confinamiento, es importante aportar programas de actividad física para mejorar la salud mental de las jugadoras de baloncesto, las cuales están acostumbradas a una práctica de actividad física regular.

Palabras Clave: Actividad Física; Baloncesto; Gamificación; Estado de Ánimo; Confinamiento.

\begin{abstract}
The main objective of this research is to analyze the effect on the mood of female basketball players, through the practice of gamified physical activity during the confinement stage due to COVID-19. The sample used was 26 players from three different categories: U14, U18 and senior, with an average age 16.65 (3.84) years. To analyze the effect on the players' mood, a quasi-experimental study was conducted through a pretest, an intervention and a final posttest, providing the Profile of Mood States (POMS) questionnaire before and after each session. The results obtained showed improvements in the players' mood was produced due to the practice of gamified physical activity, decreasing the values that are related to negative emotional states. During times of confinement, it is important to provide physical activity programs to improve the mental health of female basketball players, who are accustomed to practice regular physical activity.
\end{abstract}

Keywords: Physical Activity; Basketball; Gamification; Mood; Confinement.

\section{Introducción}

La crisis epidemiológica de la COVID-19 ha puesto en una situación sin precedentes a toda la población mundial, y como no, también a la sociedad española. Esta situación tan anómala ha provocado que las personas cambien hábitos y comportamientos generando nuevos paradigmas morales, culturales, sociales y deportivos (Sánchez et al., 2020).

En un esfuerzo por detener la propagación del virus, los gobiernos de todo el mundo ordenaron un aislamiento social, preventivo y obligatorio, aspecto éste que esta provocando cambios en los hábitos deportivos y de salud en la población en general (OMS, 2020). Dicho aislamiento obligatorio indicado por las autoridades sanitarias, ha demostrado su eficacia, pero también ha provocado problemas en la salud mental de los ciudadanos,

Fecha recepción: 15-01-21. Fecha de aceptación: 06-06-21

PreferenciasAlberto Rodríguez Cayetano

arodriguezca@upsa.es provocando cambios en los hábitos saludables, en la práctica de ejercicio físico y ha generado frustración y otras consecuencias negativas (Picco et al., 2020).

Por ello, surge la necesidad de utilizar nuevas metodologías de entrenamiento, como el uso de los videojuegos, implementados como elemento educativo y de entrenamiento, con un gran potencial para los docentes y entrenadores (Prensky, 2001), lo que unido a las nuevas Tecnologías de la Información y Comunicación (TIC) posibilitan nuevas oportunidades para el entrenador que deben ser aprovechadas (Martí et al., 2016), siendo una fuente de investigación sobre los usos, métodos, técnicas, efectos, etc. que tienen para los jóvenes actuales, ya que están dentro de lo que se conoce como modelo de educación 4.0 (De la Iglesia, 2019). De este modo, esta idea abre nuevas vías para que los profesionales de la enseñanza de la Educación Física y del entrenamiento deportivo, desarrollen propuestas didácticas que faciliten, no sólo seguir con las clases de Educación Física, sino la realización de actividad física con intensidad moderada en casa, cuando las condicio- 
nes obligan al alumnado a permanecer confinados en casa (Burgueño et al., 2020).

Una forma metodológica novedosa es lo que se conoce como gamificación, que es definida por Gómez (2020) como: «el uso, proceso, enfoque, práctica y/o experiencia que toma elementos y principios del diseño de juegos en contextos que no son de juego» (p. 14). Se considera como una estrategia metodológica novedosa en el área de las Ciencias del Deporte y de la Educación Física (Escaravajal \& Martín-Acosta, 2019; Fernández-Rio et al., 2020).

También se le conoce como exergaming, propio de los vocablos exercise y game o videojuego activo, donde se combinan el juego y ejercicio físico (Agreda et al., 2016), y para otros investigadores como gamificación (Teixes, 2005). Se utiliza para trabajar la condición física o las habilidades motrices (Marco-Ahulló et al., 2019) e integra a la vez los videojuegos con otros elementos del entrenamiento y la formación, pasando el jugador a ser el centro del aprendizaje (Escaravajal \& MartínAcosta, 2019) o del entrenamiento, realizando una dinámica totalmente diferente a los modelos tradicionales de entrenamiento. De esta forma, el entrenadordocente debe actuar como guía para conseguir ser motivador de los deportistas, utilizando la tecnología como medio de entrenamiento y enseñanza deportiva (Martín \& Faya, 2016; Prensky, 2013) y con ello, dejar atrás los planteamientos más tradicionales que ha tenido el entrenamiento deportivo, generando nuevas formas de entrenar, enseñar y motivar (Agreda et al., 2016; De la Iglesia, 2019; Fernández-Río et al., 2020).

Se trata, por lo tanto, de llevar a cabo el proceso de entrenamiento deportivo de una forma más motivante y, a la vez, ser capaz de mejorar la condición física utilizando las TIC de forma novedosa y lúdica (Agreda et al., 2016; Alsawaier, 2018; Castro-Sánchez et al., 2015; McNarry \& Mackintosh, 2016; Pérez-López et al., 2017), ayudando esta dinámica a mejorar la condición física (Agreda et al., 2016; McNarry \& Mackintosh, 2016) y a reducir las emociones negativas que pueden provocar las tareas deportivas (Lavega et al., 2013). Las TIC aplicadas al contexto deportivo, tienen el potencial para ser medios oportunos que hagan frente al reto y los desafíos de responder a las necesidades de la educación a distancia, y particularmente desde el confinamiento por COVID-19, al explorar y hacer uso de los diferentes recursos digitales aplicados a la actividad física y, en este caso, a la educación para combatir el sedentarismo desde la Educación Física (Arras et al., 2020).

Las situaciones emocionales por las que pasan los deportistas están relacionadas con la forma en la que responden ante los distintos sucesos que le acontecen (Castañeda et al., 2008). El estado de ánimo es un estado emotivo general que actúa sobre la persona y es provocado por los acontecimientos que cada uno percibe y que, dependiendo de cada sujeto, tienen una duración variable, influyendo en los procesos cognitivos propios de cada uno (Reigal \& Videra, 2013). Determina cómo nos sentimos y en función de ello, nuestra actuación posterior, ya sea de forma positiva o negativa (Castañeda et al., 2018).

El estado de ánimo y el efecto que produce la actividad física ha sido estudiado tanto de forma prolongada como de forma aguda (Reigal \& Videra, 2013; Souza et al., 2015) y se sabe que mejora el bienestar, como señala la teoría de la distracción (Arruza et al., 2008), si bien, el efecto puede ser diferente en función del tipo de carga que se lleva a cabo (Mack et al., 2000; Souza et al., 2015). En esta misma línea de investigación, la realización de actividades deportivas que sean agradables, voluntarias, gratificantes y con participación grupal, generan mejores y mayores beneficios que las que son competitivas, intensas y con alta exigencia (Díaz, 2004; Peluso \& Andrade, 2005; Reigal \&Videra, 2013), creando a su vez, una mejora en las relaciones con los demás (Reigal \& Videra, 2013) al contrario que aquellas en las que el estrés sea elevado el resultado sobre el estado de ánimo puede ser negativo (Rehor et al., 2001).

Actualmente, aunque cada vez la brecha es menor, las mujeres muestran las numerosas dificultades y barreras que siguen teniendo en la participación para la práctica deportiva, que afectan a aspectos biológicos, educativos, culturales, psicológicos y, sobre todo, deportivos (Sanz, 2017). A pesar de los beneficios que ofrece el deporte, existen notables diferencias en la participación en el deporte relacionadas con el género, siendo muy inferior a los hombres en el caso de las mujeres (Flores, 2020). Luis-De Cos et al. (2019) mostraron como un mayor nivel de competencia motriz en las mujeres podría influir positivamente en aspectos psicosociales reduciendo factores como la ansiedad en mujeres adolescentes de 14 años.

Este hecho, induce a pensar que se debe seguir investigando con el fin de apoyar a las mujeres para que las diferencias deportivas entre hombres y mujeres se reduzcan) y aportando nuevas investigaciones sobre el género femenino en el ámbito deportivo (Alfaro, 2004).

Por ello, el objetivo principal de la investigación es analizar el efecto de la práctica de actividad gamificada sobre el estado de ánimo de jugadoras de baloncesto en 
etapa de confinamiento, provocado por la COVID-19. En segundo lugar, se pretende estudiar los resultados obtenidos en función de la categoría en la que compiten las jugadoras, el nivel de preocupación hacia la problemática de la COVID-19, los días de confinamiento y el nivel de práctica de actividad física.

\section{Material y métodos}

La investigación que se realiza es de tipo cuantitativa cuasi-experimental a través de un estudio de campo con un pretest, intervención y un postest final, para comprobar el efecto de la intervención sobre el estado de ánimo de las jugadoras de baloncesto.

\section{Muestra}

La muestra está formada por 26 jugadoras de baloncesto de tres categorías diferentes: infantil, juvenil y senior, con una media de edad de 16.65 (士3.84) años. Por categorías en la que compitieron durante la temporada 2019/2020, seis jugadoras pertenecían a la categoría infantil, diez a la categoría junior y otras diez a la categoría senior. En función de la preocupación hacia la situación generada por la COVID-19, el 26.92\% $(n=7)$ de las jugadoras mostraron una preocupación moderada mientras el 73.08\% $(n=19)$ presentaron una preocupación máxima. Por otro lado, en función de los días sin salir de casa, tres de las jugadoras llevaban sin salir de casa menos de diez días, mientras que 23 de ellas llevaban más de diez días confinadas sin salir de casa. Con respecto a la práctica de actividad física semanal antes del confinamiento, diez jugadoras practicaban entre una y cuatro horas semanales, once de ellas, lo hacían entre cinco y seis horas, y sólo tres de ellas practicaban más de seis horas semanales. Por último, con respecto a la práctica de actividad física durante las semanas de confinamiento, catorce jugadoras confirmaron que practicaron menos actividad física de lo que acostumbraban, mientras que seis jugadoras afirmaron practicar las mismas horas de actividad física y otras seis, que hacían más de lo que solían realizar.

\section{Instrumento de medida}

El instrumento utilizado para la realización de esta investigación fue el Profile of Mood States (POMS) elaborado por McNair et al. (1971) en la versión reducida en castellano compuesta por 30 ítems, realizado por Andrade, et al. (2013) con valores de consistencia interna entre .78 y .88 , expresados mediante el coeficiente alfa de Cronbach. Este cuestionario presenta seis factores que miden: Cólera, Fatiga, Vigor, Amistad, Tensión y Estado Deprimido. La escala de medida es de tipo Likert con un rango de cero a cuatro, siendo $0=$ nada $1=$ un poco $2=$ moderado $3=$ bastante y $4=$ muchísimo. Se realizó antes y después de cada una de las cinco sesiones de trabajo realizadas, con un total de 130 respuestas.

\section{Procedimiento}

La recogida de datos se ha realizado durante el periodo de confinamiento provocado por la COVID-19, durante la tercera de semana de confinamiento en España, es decir, desde el lunes 30 de marzo hasta el viernes 03 de abril, ambos inclusive. En primer lugar, se procedió a contactar con los responsables del equipo deportivo al que pertenecían los jugadores. Una vez obtenido el visto bueno, se programó con los entrenadores de los equipos las sesiones diarias de entrenamiento que tendrían que realizar las jugadoras para la mejora de su condición física, así como se pidió un consentimiento informado a todas las deportistas, o a los padres/madres de las jugadoras, en el caso de los deportistas menores de edad, cumpliéndose la Declaración Ética de Helsinki en todos sus términos (2013). El proceso de gamificación comenzó el domingo 29 de marzo. Cada una de las jugadoras, recibió en su correo electrónico un vídeo creado con la aplicación IMovie en el que se les retaba a vencer a un villano, en este caso, a su entrenador. Para conseguir la victoria, debían realizar actividad física de forma conjunta, es decir, completar los retos de cada una de las cinco sesiones que tendrían a lo largo de los días siguientes, convirtiéndose en los superhéroes del baloncesto español. Para comprobar la veracidad de la ejecución de los retos motrices, se concretó un horario en el que las jugadoras debían hacer el trabajo, bajo la supervisión de los investigadores. Al finalizar cada una de ellas, se estableció una clasificación en la que se sumaban los puntos obtenidos por cada uno de los equipos, tras la consecución de los retos.

\section{Análisis estadístico}

Para el análisis de los datos, se calcularon los descriptivos principales (media y desviación típica) antes y después de su realización, tanto a nivel general, como en función de las variables, la preocupación por la COVID-19, en función de los días sin salir de casa, las horas de práctica de actividad físico/deportiva semanales antes del confinamiento y las horas de actividad física que realizaban durante el confinamiento. Posteriormente, se utilizaron pruebas paramétricas (prueba T de 
Student para muestras relacionadas) para estudiar si había diferencias en los factores del estado de ánimo antes y después de la realización de la actividad física gamificada, en función de las variables anteriormente descritas. Por último, se analizaron las correlaciones bivariadas de Peason para determinar las relaciones entre factores. Para todo ello, se ha utilizado el paquete estadísticos SPSS para Windows v.20.

\section{Resultados}

En primer lugar, se realiza un análisis de los resultados descriptivos principales de los factores que miden el estado de ánimo de las jugadoras, antes y después de la realización de la actividad física gamificada. Existe un descenso en los valores obtenidos en los factores cólera y depresión, y un aumento del nivel de fatiga y vigor, con diferencias significativas en los cuatro factores $(p<$ .05) (Tabla 1).

Con respecto a la categoría en la que compiten, existen diferencias significativas en los factores cólera y fatiga en todas las jugadoras $(p<.05)$, con una disminución del nivel del primer factor y un aumento del segundo de ellos. Por otra parte, existen diferencias significativas $(p<.05)$ en el factor depresión, tanto en categoría juvenil como en senior, disminuyendo su valor. Por último, existe un aumento significativo $(p<.05)$ del nivel de fatiga y vigor en las jugadoras que compiten en categoría senior (Tabla 2).

En función de los resultados obtenidos por la preocupación por la situación provocada por la COVID-19, se pueden observar diferencias significativas entre el pretest y postest en todos los factores, excepto en el factor tensión, entre las jugadoras que tienen una preocupación máxima sobre esta situación (Tabla 3).

Respecto a los resultados obtenidos en función de los días sin salir de casa debido al confinamiento, se puede observar cómo las jugadoras que llevan sin salir de casa menos de 10 días obtienen diferencias significativas $(p<.05)$ en los factores cólera y vigor, mientras que las jugadoras que llevan sin salir de casa más de 10 días no sólo obtienen diferencias significativas en esos dos factores, sino también en los factores vigor y depresión (Tabla 4).

En función del número de horas de actividad física que las jugadoras realizan en una semana habitual, se observa como las jugadoras que practican entre una y cuatro horas a la semana y las que lo hacen entre cinco y seis horas, muestran diferencias significativas en los mismos factores: cólera, fatiga, vigor y depresión. Por otro lado, respecto a las deportistas que dedican más de seis horas semanales a practicar actividad física y/o deportiva, sólo se observan diferencias significativas en el

Tabla 1

\begin{tabular}{|c|c|c|c|c|c|}
\hline & \multicolumn{2}{|c|}{ Pretest } & \multicolumn{2}{|c|}{ Postest } & \multirow[b]{2}{*}{ Sig } \\
\hline & Media & DT & Media & DT & \\
\hline Cólera & 2.05 & 1.02 & 1.52 & .74 & $.000 *$ \\
\hline Fatiga & 2.03 & .92 & 2.70 & .84 & $.000 *$ \\
\hline Vigor & 2.80 & 1.03 & 3.29 & .77 & $.000 *$ \\
\hline Amistad & 3.50 & .96 & 3.71 & .84 & .057 \\
\hline Tensión & 2.22 & 1.01 & 2.26 & .94 & .762 \\
\hline Depresión & 1.90 & .84 & 1.57 & .66 & $.000 *$ \\
\hline
\end{tabular}
$* \mathrm{p}<.05$

Tabla 2 .

Resultados descriptivos generales en el estado de ánimo de las jugadoras en función de la categoría en la que conpten durante b tempor d 2019/2020

\begin{tabular}{|c|c|c|c|c|c|c|c|c|c|}
\hline & \multicolumn{3}{|c|}{ Infantil } & \multicolumn{3}{|c|}{ Juvenil } & \multicolumn{3}{|c|}{ Senior } \\
\hline & Pretest & Postest & & Pretest & Postest & & Pretest & Postest & $\mathrm{Sig}$ \\
\hline & Media & Media & & Media & Media & & Media & Media & \\
\hline Cólera & 1.79 & 1.29 & $.019 *$ & 1.80 & 1.42 & $.004 *$ & 2.46 & 1.76 & $.001 *$ \\
\hline Fatiga & 1.68 & 2.19 & $.035 *$ & 2.05 & 2.85 & $.000 *$ & 2.23 & 2.88 & $.000 *$ \\
\hline Vigor & 3.10 & 3.26 & .534 & 2.91 & 3.22 & .104 & 2.51 & 3.37 & $.000 *$ \\
\hline Amistad & 3.47 & 3.57 & .738 & 3.74 & 3.88 & .407 & 3.27 & 3.62 & $.025 *$ \\
\hline Tensión & 2.18 & 2.05 & .645 & 2.10 & 2.14 & .818 & 2.38 & 2.51 & .432 \\
\hline Depresión & 1.61 & 1.34 & $.129 *$ & 1.65 & 1.42 & $.027 *$ & 2.33 & 1.86 & $.007 *$ \\
\hline
\end{tabular}

Tabla 3.

Resultados descriptivos generales en el estado de ánimo de las jugadoras en función de su preocupación por la COVID -19

\begin{tabular}{|c|c|c|c|c|c|c|c|c|c|c|}
\hline & \multicolumn{5}{|c|}{ Moderada } & \multicolumn{5}{|c|}{ Máxima } \\
\hline & Pret & & Pos & & & & & & & \\
\hline & Media & DT & Media & DT & Sig & Media & DT & Media & DT & Sig \\
\hline Cólera & 1.59 & .74 & 1.34 & .59 & .115 & 2.24 & 1.06 & 1.59 & .78 & $.000 *$ \\
\hline Fatiga & 1.66 & .70 & 2.30 & 1.00 & $.002 *$ & 2.18 & .96 & 2.86 & .72 & $.000 *$ \\
\hline Vigor & 3.44 & .91 & 3.41 & .95 & .881 & 2.55 & .97 & 3.24 & .70 & $.000 *$ \\
\hline Amistad & 3.78 & .99 & 3.79 & 1.05 & .964 & 3.38 & .93 & 3.67 & .74 & $.018^{*}$ \\
\hline Tensión & 1.98 & 1.13 & 1.99 & 1.08 & .967 & 2.32 & .94 & 2.37 & .86 & .725 \\
\hline Depresión & 1.42 & .57 & 1.32 & .47 & .429 & 2.09 & .85 & 1.67 & .70 & $.000 *$ \\
\hline
\end{tabular}

${ }^{*} \mathrm{p}<.05$

Tabla 4 .

Resultados descriptivos generales en el estado de ánimo de las jugadoras en función de los días sin salir de casa

\begin{tabular}{ccccccc}
\hline \multicolumn{7}{c}{ Días sin salir de casa } \\
\hline & \multicolumn{5}{c}{ Entre 0 y 10 días } & \multicolumn{3}{c}{ Más de 10 días } \\
\hline Cólera & 2.64 & 1.71 & $.032^{*}$ & Pretest & Postest & Sig \\
\hline Fatiga & 2.63 & 2.73 & .770 & 1.93 & 1.49 & $.000^{*}$ \\
Vigor & 2.34 & 3.53 & $.000^{*}$ & 2.88 & 3.70 & $.000^{*}$ \\
Amistad & 3.40 & 3.79 & .200 & 3.51 & $.003 *$ \\
Tensión & 1.91 & 1.81 & .617 & 2.28 & 2.32 & .125 \\
Depresión & 2.13 & 1.70 & .152 & 1.86 & 1.55 & $.002 *$ \\
\hline *p $<.05$ & & & & & &
\end{tabular}

Tabla 5 .

Resultados descriptivos en función de las horas de Actividad física en una semana habitual

\begin{tabular}{|c|c|c|c|c|c|c|c|c|c|}
\hline \multicolumn{10}{|c|}{ Horas Actividad física semana habitual } \\
\hline & \multicolumn{2}{|c|}{ Entre 1 y 4 horas } & \multirow{2}{*}{ Sig. } & \multicolumn{2}{|c|}{ Entre 5 y 6 horas } & \multirow{2}{*}{ Sig. } & \multicolumn{2}{|c|}{ Más de 6 horas } & \multirow{2}{*}{ Sig. } \\
\hline & Pre & Post & & Pre & Post & & Pre & Post & \\
\hline Cólera & 2.12 & 1.53 & $.002 *$ & 2.04 & 1.49 & $.000 *$ & 1.82 & 1.60 & .465 \\
\hline Fatiga & 1.99 & 2.78 & $.000 *$ & 2.10 & 2.59 & $.003 *$ & 1.92 & 2.91 & $.007 *$ \\
\hline Vigor & 2.68 & 3.22 & $.001 *$ & 2.77 & 3.35 & $.001 *$ & 3.53 & 3.31 & .579 \\
\hline Amistad & 3.28 & 3.54 & .121 & 3.69 & 3.80 & .478 & 3.58 & 3.93 & .387 \\
\hline Tensión & 2.05 & 2.10 & .725 & 2.33 & 2.22 & .524 & 2.58 & 2.99 & .400 \\
\hline Depresión & 1.99 & 1.61 & $.017 *$ & 1.78 & 1.47 & $.011 *$ & 2.05 & 1.81 & .424 \\
\hline
\end{tabular}
\begin{tabular}{llllllllll} 
Depresión & 1.99 & 1.61 & $.017 *$ & 1.78 & 1.47 & $.011 *$ & 2.05 & 1.81 & 424 \\
\hline
\end{tabular} ${ }^{*} \mathrm{p}<.05$

Tabla 6.

Resultados descriptivos en función del nivel de Actividad física

\begin{tabular}{|c|c|c|c|c|c|c|c|c|c|}
\hline \multicolumn{10}{|c|}{ Nivel de Actividad Física } \\
\hline & \multicolumn{3}{|c|}{ Más horas } & \multicolumn{3}{|c|}{ Mismas horas } & \multicolumn{3}{|c|}{ Menos horas } \\
\hline & Pretest & Postest & \multirow{2}{*}{ Sig. } & Pretest & Postest & \multirow{2}{*}{ Sig. } & Pretest & Postest & \multirow{2}{*}{ Sig. } \\
\hline & Media & Media & & Media & Media & & Media & Medía & \\
\hline Cólera & 1.79 & 1.48 & .133 & 2.12 & 1.67 & $.036 *$ & 2.14 & 1.48 & $.000 *$ \\
\hline Fatiga & 1.90 & 2.91 & $.000 *$ & 2.43 & 2.83 & .082 & 1.92 & 2.55 & $.000 *$ \\
\hline Vigor & 2.95 & 3.32 & .184 & 3.05 & 3.31 & .228 & 2.63 & 3.27 & $.000 *$ \\
\hline Amistad & 3.60 & 3.94 & .116 & 3.55 & 3.53 & .901 & 3.43 & 3.67 & .149 \\
\hline Tensión & 2.28 & 2.48 & .453 & 2.43 & 2.30 & .609 & 2.11 & 2.14 & .863 \\
\hline Depresión & 1.70 & 1.51 & .260 & 2.10 & 1.87 & .295 & 1.90 & 1.48 & $.001 *$ \\
\hline
\end{tabular}

Tabla 7.

Correlaciones bivariadas de los factores del estado de ánimo

\begin{tabular}{|c|c|c|c|c|c|c|}
\hline & Cólera & Fatiga & Vigor & Amistad & Tensión & Depresión \\
\hline Cólera & 1 & $.399 * *$ & $-.363 * *$ & $-.453 * *$ & $.273 * *$ & $.774 * *$ \\
\hline Fatiga & $.399 * *$ & 1 & $-.211 * *$ & $-.187 * *$ & $.296 * *$ & $.390 * *$ \\
\hline Vigor & $-.363 * *$ & $-.211 * *$ & 1 & $.543 * *$ & $.133 *$ & $-.307 * *$ \\
\hline Amistad & $-.453 * *$ & $-.187 * *$ & $.543 * *$ & 1 & .018 & $-.432 * *$ \\
\hline Tensión & $.273 * *$ & $.296 * *$ & $.133 *$ & .018 & 1 & $.434 * *$ \\
\hline Depresión & $.774 * *$ & $.390 * *$ & $-.307 * *$ & $-.432 * *$ & $.434 * *$ & 1 \\
\hline
\end{tabular}


factor fatiga (Tabla 5).

Respecto a los resultados obtenidos según el nivel de horas de práctica de actividad física en el periodo de confinamiento, se puede observar cómo las jugadoras que practican menos horas de actividad físico-deportiva de lo que acostumbran, presentan diferencias significativas $(p<.05)$ en los factores cólera, fatiga, vigor y depresión (Tabla 6).

Por último, se muestran las correlaciones entre todos los factores que conforman el estado de ánimo de las jugadoras de baloncesto (Tabla 7). Para el factor cólera, se puede observar cómo existe una correlación significativa positiva entre este factor y la fatiga, tensión y depresión de las jugadoras, y una correlación significativa negativa con los factores vigor y amistad. En el factor fatiga, se observa una correlación significativa positiva con los factores cólera, tensión y depresión, y una correlación significativa negativa entre con los factores vigor y amistad. Respecto al factor vigor, obtiene una correlación significativa positiva con el factor amistad y una correlación positiva significante con el factor tensión, mientras que, se observa una correlación significativa negativa entre este factor y las variables cólera, fatiga y depresión. Siguiendo con el factor amistad, se observa una correlación significativa positiva con el factor vigor, mientras que, se observa una correlación negativa significativa con los factores cólera, fatiga y depresión. Para el factor tensión, se producen correlaciones significativas positivas con los factores cólera, fatiga, vigor y depresión. Por último, en el factor depresión se observan correlaciones significativas positivas con los factores cólera, fatiga y tensión, mientras que se obtienen correlaciones significativas negativas entre el factor depresión y los factores vigor y amistad (Tabla 7).

\section{Discusión}

A nivel general, los resultados obtenidos mostraron cómo se produjo una mejora en el estado de ánimo de las jugadoras de baloncesto debido a la práctica de actividad física gamificada, disminuyendo los valores que se relacionan con estados emocionales negativos (Lavega et al., 2013), a excepción del factor tensión que muestra valores similares, mientras que se produjo un aumento del factor Vigor y Amistad, relacionado con un estado emocional positivo. Estos resultados van en la línea de los trabajos realizados por Fernández-Rio et al. (2020), McLafferty et al. (2004), Reigal \& Videra (2013) y Taylor-Piliae et al. (2006). Sin embargo, no coinciden con las investigaciones que manifiestan que las mujeres descienden sus valores positivos y muestran mayores factores negativos (Arruza et al., 2008; Chacón et al., 2017; Goikoetxea, et al., 2014).

Estos datos pueden reforzar la idea de considerar la gamificación como un recurso metodológico muy útil para la fidelización de las personas en la práctica de actividad física (Almirall, 2016; Mujica \& Jiménez, 2020; Navarro et al., 2017). Además, las dinámicas relacionadas con la gamificación, con tareas de carácter cooperativo o de equipo (Deif, 2017; Pérez-López et al., 2017a), tienen una mayor probabilidad de conseguir este tipo de mejoras, que no tareas con un mayor nivel de exigencia e intensidad (Peluso \& Andrade, 2005). Se está de acuerdo con el estudio realizado por AntónCandanedo y Fernández Río (2017) al considerar que las dinámicas de juego que requieren la experiencia gamificada de un modo cooperativo, tienen una mayor probabilidad de conseguir una mayor mejora en estos factores positivos, como es en nuestro estudio.

Por otro lado, diversos estudios han demostrado como la mejora en el estado de ánimo de las personas, no sólo tiene efectos directos sobre este factor, sino que ayuda a generar pensamientos más reflexivos, consiguiendo una mejor adaptación a la situación y a la realidad del entorno (Fernández-Abascal et al., 2003; Lyubomirsky et al., 2005; Reigal et al., 2013). Este hecho es esencial en la sociedad actual, en una etapa de incertidumbre absoluta y de constante adaptabilidad a situaciones excepcionales.

La preocupación por la COVID-19 hace que, cuanto mayor es la preocupación que tienen las jugadoras sobre esta situación, aumenta el valor asociado a emociones negativas del estado de ánimo y disminuye el nivel de los factores positivos. Al ser una situación excepcional, no existen estudios acordes a esta línea de investigación que se propone.

\section{Conclusión}

El objetivo principal de esta investigación fue analizar el efecto de la práctica de actividad física gamificada sobre el estado de ánimo de jugadoras de baloncesto durante la etapa de confinamiento provocado por la COVID-19. Una intervención de práctica de actividad física gamificada ha mejorado el estado de ánimo de jugadoras de baloncesto durante la etapa de confinamiento, por lo que, es muy recomendable proponer actividades de promoción de actividad física y deportiva, utilizando nuevos recursos metodológicos como la 
gamificación, buscando una mejora de la condición física y de ocupación del ocio y tiempo libre en la sociedad, para que las personas mejoren su estado de ánimo.

En segundo lugar, se pretendió examinar los resultados obtenidos en función de la categoría en la que competían las jugadoras, el nivel de preocupación hacia la problemática del COVID-19, los días de confinamiento y el nivel de práctica de actividad física. Para este objetivo, es importante destacar que si se quiere mejorar el estado de ánimo de las deportistas que están acostumbrados a la práctica de actividad físico-deportiva de una forma regular y, de repente, debido a una situación de este tipo, no pueden continuar con su actividad, es imprescindible la realización de programas de actividad física, adaptados a las características personales de cada sujeto, con la ayuda de las TIC, y así, poder aumentar el vigor y la amistad de esos sujetos, y así a su vez, disminuir las emociones negativas que puedan provocar un estado depresivo, así como tener en cuenta las necesidades psicológicas de cada una de ellas.

Se considera pertinente realizar nuevas indagaciones que puedan enfocarse, de forma más específica, en la mejora del estado de ánimo y el mantenimiento a lo largo del tiempo. Entre aquellos aspectos, destacaría los siguientes: a) comparar entre jugadores y jugadoras de baloncesto; b) relacionar el estado de ánimo con el nivel del compromiso de las jugadoras hacia el deporte; y c) examinar el efecto sobre el estado de ánimo de la práctica de actividad física gamificada y la monotonía de la realización de tareas técnicas aisladas.

\section{Referencias}

Agreda, M., Raso, F. \& Hinojo-Lucena, F.J. (2016).TendenciasTIC para la innovación en educación física: el exergaming como alternativa complementaria a la clase tradicional. Trances, 81 , 311-320.

Alfaro, É. (2004). El talento psicomotor y las mujeres en el deporte de alta competición. Revista de Educación física, (335), 127 151.

Almirall, L. (2016). Epic clans. Gamificando la Educación Física. RevistaTándem, (51), 67-73.

Alsawaier, R. S. (2018). The effect of gamification on motivation and engagement. International Journal of Information and Learning Technology, 35(1), 56-79. https://doi.org/10.1108/ IJILT-02-2017-0009.

Andrade, E., Arce, C., De Francisco, C., Torrado, J. \& Garrido, J. (2013).Versión breve en español del cuestionario POMS para deportistas adultos y población general. Revista de Psicología del Deporte, 22(1), 95-102.

Anton-Candanedo, A. \& Fernandez-Rio, J. (2017). Hibridando modelos pedagogicos para la mejora de la comprension tactica de estudiantes: una investigacion a traves del Duni. Agora para la Educacion Fisica y el Deporte 19(2-3), 257- 276. https:// doi.org/10.24197/aefd.2-3.2017.257-276.

Arras-Vota, A., Bordas-Beltrán, J., Mondaca-Fernández, F., \& Rivera-Sosa, J. (2020). El caso sede México: Formación en Educación Física en e-entornos universitarios durante la contingencia de la COVID-19 (The México heartquarters case: Physical Education Teacher Education in e-university environments during the COVID-19 contingency). Retos, 41, 35-46. https://doi.org/10.47197/retos.v0i41.83529

Arruza, J.A.,Arribas, S., Gil, L., Irazusta, S., Romero, S. \& Cecchini, J.A. (2008). Repercusiones de la duración de la actividad físicodeportiva sobre el bienestar psicológico. Revista Internacional de Medicina y Ciencias de la Actividad Física y el Deporte, 8(30), 171-183. https://doi.org/10.24310/riccafd.2020.v9i1.8302. Burgueño, R., Bonet-Medina,A., Cerván-Cantón, Álvaro, Espejo, R., Fernández-Berguillo, F., Gordo-Ruiz, F., LinaresMartínez, H., Montenegro-Lozano, S., Ordoñez-Tejero, N., Vergara-Luque, J., \& Gil-Espinosa, F. (2020). Educación Física en Casa de Calidad. Propuesta de aplicación curricular en Educación Secundaria Obligatoria (Quality Physical Education at Home. Curricular implementation proposal in Middle Secondary School). Retos, 39, 787-793. https://doi.org/ 10.47197/retos.v0i39.78792

Castañeda, C.; Zagalaz, M. L.; Arufe, V.; Campos-Mesa, Mª C. (2018). Motivos hacia la práctica de actividad física de los estudiantes universitarios. Revista Iberoamericana de Psicología del Ejercicio y el Deporte, 13(1), 79-89. http://dx.doi.org/ 10.15446/revfacmed.v64n2.53068.

Castro-Sanchez, M., Espejo-Garces, T., Valdivia-Moral, P., ZuritaOrtega, F., Chacon-Cuberos, R. \& Cabrera-Fernandez, A. (2015). Importancia de los exergames en la educacion fisicodeportiva. Trances, 75, 657-676.

Chacón, F., Corral, J.A. \& Castañeda, C. (2017). Relación entre actividad física, estado de ánimo y género en personas adultas. European Journal of Health Research, 3(3), 163-171. https: / / doi.org/10.30552/ejhr.v3i3.73

De la Iglesia, $\mathrm{M}^{\mathrm{a}}$. C. (2019). Caja de herramientas 4.0 para el docente en la era de la evaluación por competencias. Innovación Educativa, 19(80), 93-112. https: / / doi.org/10.26754/ cinaic. 2019.0034

Díaz, M.P. (2004). La actividad física deportiva: más allá de lo saludable. Revista de la Facultad de Medicina de la Universidad Nacional de Colombia, 52(3), 212-221. https://doi.org/ 10.25176/rfmh.v16.n1.344

Deif,A. (2017). International Journal of Lean Six Sigma Insights on lean gamification for higher education. International Journal of Lean Six Sigma, 8(3), 359-376. https: / /doi.org/10.1108/ijlss04-2016-0017

Escaravajal, J.C. \& Martín-Acosta, F. (2019). Análisis bibliográfico de la gamificación en Educación Física. Revista Iberoamericana de Ciencias de la Actividad Física y el Deporte, 8(1), 97-109. https: / /doi.org/10.24310/riccafd.2019.v8i1.5770

Fernández-Abascal, E., Jiménez, M., \& Martín, M. (2003). Emoción y Motivación: la adaptación humana. Madrid: Centro de Estudios Ramón Areces, SA.

Fernández-Rio, J., las Heras, E., González,T., Trillo,V. \& Palomares, J. (2020). Gamification and Physical Education:Viability and preliminary views from students and teachers. Physical 
Education and Sport Pedagogy, 25(5), 509-524. https://doi.org/ 10.1080/17408989.2020.1743252

Flores, Z. (2020). Mujer y deporte en México: hacia una igualdad sustancial. Retos: nuevas tendencias en educación física, deporte y recreación, (37), 222-226. https://doi.org/10.47197/ retos.v37i37.71684

Goikoetxea, A. O., Martínez, R., Usabiaga, O., Etxebeste, J. \& Urdangarin, C. (2014). Efecto de los juegos de cooperaciónoposición en el ánimo de los universitarios. Retos, 25, 58-62. https://doi.org/10.47197/retos.v0i25.34478

Gómez, J. L. (2020). Gamificación en contextos educativos: análisis de aplicación en un programa de contaduría pública a distancia. Revista Universidad y Empresa, 22(38), 8-39. https: / /doi.org/ 10.12804/revistas.urosario.edu.co/empresa7a.6939

Lavega, P., March, J. \& Filella, G. (2013). Juegos deportivos y emociones. Propiedades psicometricas de la escala GES para ser aplicada en la Educacion Fisica y el Deporte. Revista de Investigacion Educativa, 311, 151-165. https://doi.org/ 10.6018/rie.31.1.147821

Luis-de Cos, G., Arribas-Galarraga, S., Luis-de Cos, I., \& Arruza Gabilondo, J.A. (2019). Competencia motriz, compromiso y ansiedad de las chicas en Educación Física (Motor competence, commitment, and anxiety in girls during physical education classes). Retos, 36, 231-238. https://doi.org/10.47197/ retos.v36i36.64243

Lyubomirsky, S., King, L., \& Diener, E. (2005). The benefits of frequent positive affect: Does happiness lead to success? Psychological Bulletin, 131(6), 803-855. https://doi.org/ 10.1037/0033-2909.131.6.803

Mack, M.G., Huddleston, S., Dutler, K.E. \& Bian,W.(2000). Mood state changes of students enrolled in physical activity clases. Perceptual and Motor Skills, 90, 911-914. https://doi.org/ 10.2466/pms.2000.90.3.911

McLafferty, C., Wetzstein, C., \& Hunter, G. (2004). Resistance training is associated with improved mood in healthy older adults. Perceptual and Motor Skills, 93(3), 947-957. https: / / doi.org/10.2466/pms.98.3.947-957

Marco-Ahullo, Estevan, I., Diaz-Barahora, J. \& Garcia-Masso, X. 2019. Las TAC como herramienta para mejorar el aprendizaje de conceptos de didactica de la educacion fisica en alumnado universitario. En XIII Congreso Internacional FEADEF sobre la ensenanza de la Educacion Fisica y el deporte escolar y II congreso Red Global Sevilla.

Martí, J., Méndez, E., Queiro, C., Sánchez,A., Galbis, A. \& Seguí, D. (2016). Gamificación en el ámbito universitario. Ingénesis Books.

Martín, A. \& Faya, F. (2016). El juego en el aula de lengua inglesa para consolidar contenidos: experiencia con futuros docentes de educación infantil. Nuevos enfoques didácticos, 873-878. 10.5209/DIDA.54089

Mujica, F., \& Jiménez,A. (2020). Emociones positivas del alumnado de Educación Secundaria en las prácticas de baloncesto en Educación Física (Positive emotions of the students of Secondary Education in the practices of basketball in Physical Education). Retos, 39, 556-564. https://doi.org/10.47197/ retos.v0i39.80112

McNair, D.M., Lorr, M. \& Droppleman, L.F. (1971). Profile of Mood States Manual. Educational and Industrial Testing Service. McNarry, M.A. \& Mackintosh, K.A. (2016). Investigating the
Relative Exercise Intensity of Exergames in Prepubertal Children. Games for health journal, 5(2), 135-140. https: / / doi.org/10.1089/g4h.2015.0094

Navarro, D. , Martínez, R., \& Pérez, I. (2017). El enigma de las 3 efes: fortaleza, fidelidad y felicidad. Revista Española de Educación Física y Deportes, 419, 73-85.

Peluso, M.A. \& Andrade, L.H. (2005). Physical activity and mental health: the association between exercise and mood, Clinics, 60(1), 61-70. https://doi.org/10.1590/s180759322005000100012

Pérez-López, I.J., Rivera, E. yTrigueros, C. (2017). La profecía de los elegidos: Un ejemplo de gamificación aplicado a la docencia universitaria. Revista Internacional de Medicina y Ciencias de la Actividad Física y el Deporte, 17(66), 243-260. https: / / doi.org/ 10.15366/rimcafd2017.66.003

Perez-Lopez, I. J., Rivera, E., \& Delgado-Fernandez, M. (2017a). Mejora de hábitos de vida saludables en alumnos universitarios mediante una propuesta de gamificacion. Nutricion Hospitalaria, 34(4), 942-951. https://doi.org/10.20960/nh.669

Picco J, Gonzalez Davila E, Wolff, S., Gómez,V. \&Wolff, D. (2020). Aspectos psicosociales de la pandemia de covid-19 en la población de Mendoza. Rev Arg Med, 8(2), 96-100. https: / / doi.org/ 10.7775/rac.es.v88.i3.17925

Prensky, M. (2001). Digital game-based learning. McGraw-Hill. Prensky, M. (2013). Enseñar a nativos digitales. SM Ediciones.

Rehor, P.R., Dunnagan, T., Stewart, C. \& Cooley, D. (2001). Alteration of mood state after a single bout of noncopetitive and competitive exercise programs. Perceptual and Motor Skills, 93, 249-256. https: / /doi.org/10.2466/pms.2001.93.1.249

Reigal, R. \&Videra, A. (2013). Efectos de una sesión de actividad física sobre el estado de ánimo. Revista Internacional de Medicina y Ciencias de la Actividad Física y el Deporte, 13(52), 783-798. https://doi.org/10.24310/riccafd.2018.v7i2.5103

Reigal, R., Márquez, M. V., Videra, A, Martín, I., \& Juárez, R. (2014). Efecto agudo de la actividad físicodeportiva y la expresión corporal sobre el estado de ánimo. Apunts, 113(3), 30-36. https: / / doi.org/10.5672/apunts.2014-0983.es.(2013/ 3). 113.02

Sánchez, R., Moscoso, D. \& Piedra, J. (2020). The sociology of sport in Spain: Development, current situation, and future challenges. Sport und Gesellschaft, 17(1), 69-95. https:// doi.org/10.1515/sug-2020-0004

Sanz, J.J. (2017). Una aproximación a las dificultades del deporte femenino en la alta competición: hacia la equidad e igualdad. Acción Motriz, (18), 29-36.

Souza, J.F.T.D., Giampá, S.Q.D.C., Lemos, V.D.A., Santos, S.A.D., Silva, E.T.D., De Mello, M.T., Tufik, S., Santos, R.V.T.D. \& Antunes, H.K.M. (2015). Exercise performed at hypoxia influences mood state and anxiety symptoms. Motriz Revista de Educação Física, 21(2), 177-184. https://doi.org/10.1590/ s1980-65742015000200008

Taylor-Piliae, R. E., Haskell,W. L., Waters, C. M., \& Froelicher, E. S. (2006). Change in perceived psychosocial status following a 12-week Tai Chi exercise programme. Journal of Advanced Nursing, 54(3), 313-329. https://doi.org/10.1111/j.13652648.2006.03809.x

Teixes, F. (2005). Gamificación: Fundamentos y aplicaciones. UOC. 